\title{
A wearable headset for monitoring electromyography responses within spinal surgery
}

\author{
M. R. Golab ${ }^{1}$ P. J. Breedon ${ }^{1} \cdot$ M. Vloeberghs ${ }^{2}$
}

Received: 9 May 2016/Revised: 23 May 2016/Accepted: 23 May 2016/Published online: 9 June 2016

(c) The Author(s) 2016. This article is published with open access at Springerlink.com

\begin{abstract}
Purpose This research examines an approach for enhancing the efficiency of spinal surgery utilising the technological capabilities and design functionalities of wearable headsets, in this case Google Glass. The aim was to improve the efficiency of the selective dorsal rhizotomy neurosurgical procedure initially through the use of Glass via an innovative approach to information design for an intraoperative monitoring display.

Methods Utilising primary and secondary research methods the development of a new electromyography response display for a wearable headset was undertaken.

Results Testing proved that Glass was fit for purpose and that the new intraoperative monitor design provided an example platform for the innovative intraoperative monitoring display; however, alternative wearable headsets such as the Microsoft HoloLens could also be equally viable. Conclusion The new display design combined with the appropriate wearable technology could greatly benefit the selective dorsal rhizotomy procedure.
\end{abstract}

P. J. Breedon

Philip.breedon@ntu.ac.uk

M. R. Golab

mark.golab@ntu.ac.uk

M. Vloeberghs

Michael.Vloeberghs@nuh.nhs.uk

1 Nottingham Trent University, Design for Health \& Wellbeing Research Group, Nottingham NG1 4BU, UK

2 Nottingham University Hospital Trust, Derby Road, Nottingham NG7 2UH, UK
Keywords Selective dorsal rhizotomy · Google Glass · Wearable technology $\cdot$ Intraoperative monitoring

\section{Introduction}

\section{Selective dorsal rhizotomy}

Selective dorsal rhizotomy (SDR) was first developed in 1898 , but has become more popular over the last 30 years as techniques have advanced. This is a neurosurgical procedure aimed at reducing spasticity (tight and stiff muscle tone) in the lower limbs. It is most commonly used for children with spastic diplegia (two limbs affected), which accounts for $25-30 \%$ of children born with cerebral palsy [1].

The surgery requires the bones of the spine (vertebrae) in the lower back area to be opened to reveal the conus (the end of the spinal cord) to gain access to the nerve roots Fig. 1. The traditional approach was to get to these roots through a lengthy multi-level operation gaining access to the nerves by opening several vertebrae. However, some evidence suggests the multi-level approach may have led to other spinal problems such as scoliosis (curvature of the spine). At only one level the spinal canal is opened. The lower end of the spinal cord can be identified using an ultrasound probe. The membrane covering the spinal cord, the dura, is opened and the lower end of the cord (with the sensory roots entering it) is identified. Each of the sensory nerve roots is then subdivided into three or four fascicles. Each rootlet is stimulated to identify the ones that contribute then divided. The process is repeated for all the other most to the spasticity. These rootlets are nerve roots on both sides, aiming to divide $50-70 \%$ of the sensory roots [2]. SDR focuses on nerve roots from L1 to S1 (cauda 


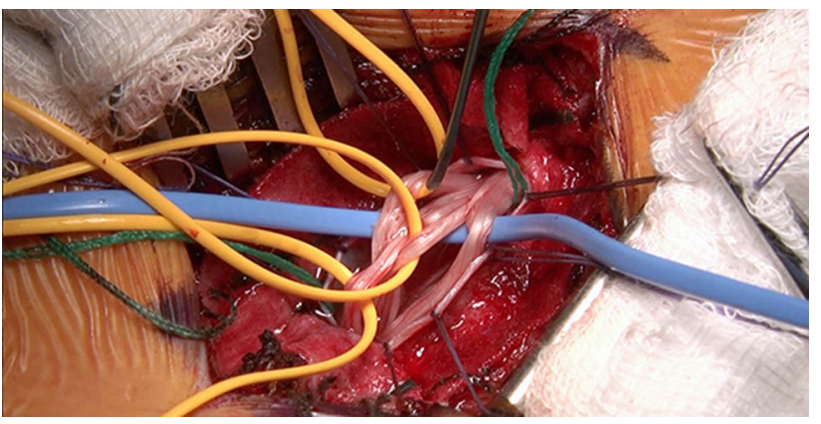

Fig. 1 Selective dorsal rhizotomy

equina/lumbar nerves) on the upper lumbar spine. After the sensory nerves are exposed, each rootlet is tested with EMG, which records electrical patterns in muscles.

During the procedure to identify the most responsive nerves, a probe is pressed against the lumbar nerves and a current is sent through them. This will establish a pure motor response sensory route. Once the probe is sending the current through the nerves the most responsive nerves will be displayed on a monitor inside the operating theatre. The monitor will show an amplitude wave, the higher fluctuation rate indicates which nerves are the most responsive. The surgeon will be trying to identify which nerves are the most responsive. This is done through intraoperative monitoring utilising electromyography.

The objectives of SDR surgery:

- Achieve a long-term reduction in spasticity.

- Improve patient function and mobility.

- Increase independence.

- Increase range of motion and improve positioning.

\section{Google Glass}

Google Glass is a wearable, voice-controlled Android device that resembles a pair of eyeglasses and displays information directly in the user's field of vision [3].

Figure 2 shows the user wearing Glass while viewing the Intraoperative display on a small projected screen positioned just above the wearers line of sight. This small but powerful screen measures $640 \times 360$ pixels. Glass responds through both tactile and vocal feedback, the use of two fingers to slide along the side of Glass lets the user navigate through the timeline. Tapping the side allows the wearer to progress through the interface whereas the sliding down motion takes the wearer back to the previous slide. On the home screen speaking the command ' $\mathrm{Ok}$ Glass' presents the user with a variety of options such as 'Take a Picture' or 'Directions to Here'. These requests can

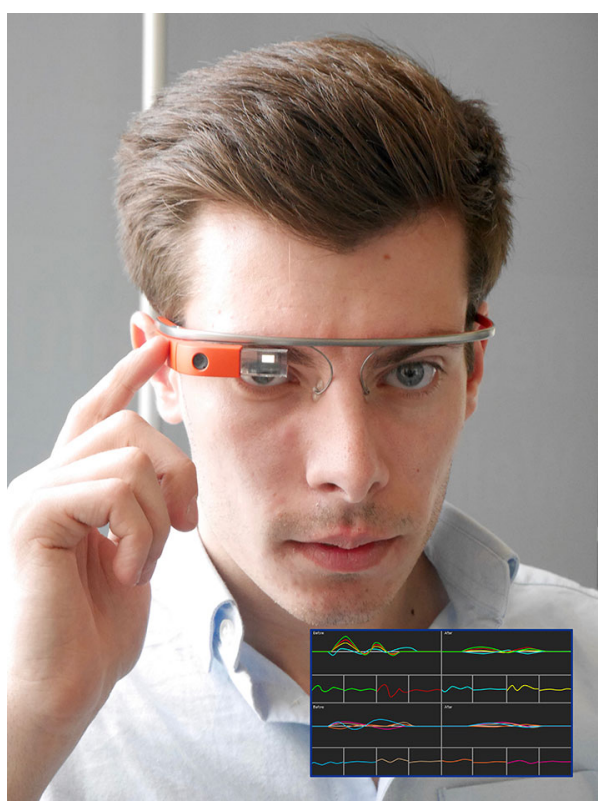

Fig. 2 Google Glass with inset showing user view of intraoperative monitor

all be activated hands free, which could be of great benefit especially during a surgical procedure.

The introduction to Glass in the workplace saw the beginning of new thinking for applying this innovative technology to improve the efficiency and success of operating procedures and address potential lack of communication. As a wearable medical device, there is the emotional value attachment, which is important in today's society. If users are not comfortable using Glass then it will not be efficient as an aid to enhance medical treatment. Val Afshar's research suggests Google Glass paints an amazing picture of how the technology is revolutionising health care. Glass provides an open canvas for application developers to shape the future health care landscape, and Google Glass is but one of the emerging wearable's transforming health care [4]. Wearable Technology has had an enormous impact on the way surgical operations are undertaken and is constantly trying to improve efficiency, raise quality and expand services. A report by the Health Research Initiative (HRI) shows that $79 \%$ of physicians and close to $50 \%$ of consumers believe using mobile devices can help physicians better coordinate care [5]. Thorough research has been undertaken into the market of wearable technology to clarify whether it is advantageous in the health care industry. From the research analysed it suggests that Google Glass as a product still has a long journey but as a concept, the technology could provide large benefits for the healthcare industry. 


\section{Method}

\section{Information design}

Information design is the planning and shaping of the contents of a message, and the environments in which it is presented with the intention to satisfy the information needs of the intended recipients [6]. It was identified that during the SDR procedure, the Neurosurgeon has to communicate with a Neurophysiologist across the operating theatre to identify which sensory nerves have to be cut. This could result in a lack of communication and efficiency, and a possibility for the surgeon to over section or under section a particular nerve. The use of Google Glass in SDR could help to resolve this issue allowing the surgeon to view the EMG responses on its display while not diminishing the concentration and focus required from the procedure. This research identified the following issues:

1. Identification of a need to design an innovative display for presenting intraoperative monitoring on Google Glass via information design;

2. Develop an understanding of research applications and product design specifications towards designing an innovative display;

3. Constant contact with the surgeon to validate the design choices and provide critical reflection throughout;

4. Follow strict medical standards throughout the design process to ensure that the system can be marketed.

Figure 3 presents a small two inch incision of the working field of view where nerve stimulation takes place. It is proposed that by utilising Google Glass it could provide significant benefit for the SDR procedure. If the surgeon was to wear Glass with all the evoked potentials being shown on the screen this would allow the surgeon to see exactly which nerves to cut without loss of concentration.

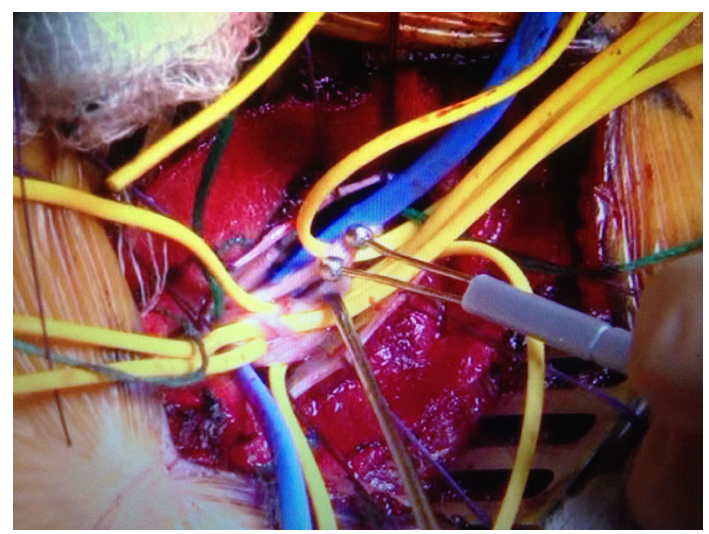

Fig. 3 Sensory nerve stimulation
If two surgeons were to wear Glass and both could see evoked potentials, this would mean that they could compare and contrast which nerves should be cut, essentially providing a second opinion.

\section{Intraoperative monitoring}

Intraoperative neurophysiologic monitoring (IONM) is a valuable technique for assessing the nervous system. It replaces the neurologic examination when the patient is under general anaesthesia [7]. SDR requires monitoring electromyography (EMG) activity generated by motor unit action potentials (MUAPs). Therefore the use of muscle paralytic agents should be minimised to perform this procedure properly. Since some institutions use pudendal somatosensory evoked potentials (SSEPs) during this procedure, it is imperative that the anaesthetic agents chosen for this surgery permit the optical recording of SSEP waveforms as well [8]. Electrophysiological methods for selectively ablating abnormal sensory nerve rootlets, thus sparing normal sensory rootlets, were proposed by Fasano et al. and later by Peacock 2-4. These methodologies came into more widespread use after standardised neurophysiologic intraoperative monitoring (NIOM) techniques were reported by Stuadt et al. [9]. Intraoperative monitoring using somatosensory evoked potentials (SSEPs) has long been accepted as a useful tool for the assessment of neurological status during spinal surgery. Even in modern practice, it is clear that monitoring does not detect all potential spinal cord damage, with the largest study, encompassing the whole gamut of spinal pathology (albeit with self-reported data) to date indicating that only $40 \%$ of post-operative cord injuries were heralded by a change in neurophysiological monitoring [10]. The implementation of new technologies such as Glass could potentially improve (IONM) techniques during surgery.

Figure 4 depicts the location of each motor nerve that is innervated with the specific muscles. For example using EMG on roots L2-L4 will give a response for the thigh adductors and quadriceps femoris. Stimulation of the nerves is performed using a constant voltage stimulator. The stimulation is performed with a square-wave pulse $0.1 \mathrm{~ms}$ in duration at $50 \mathrm{~Hz}$ for $1 \mathrm{~s}$. A $1 \mathrm{~s}$ train of $50 \mathrm{~Hz}$ square wave pulses each of $0.1 \mathrm{~ms}$ duration is given for the specific voltage selected [11]. During the procedure the S1 nerve roots are usually the first root pair to be tested. When the threshold voltage of the S1 ventral root is achieved, approximately $200 \mathrm{mV}$ should result in knee flexion and ankle planter flexion without any associated toe flexion or anal sphincter contraction. The threshold voltage should result in either a physical twitch and/or a definite EMG in the targeted muscles. 


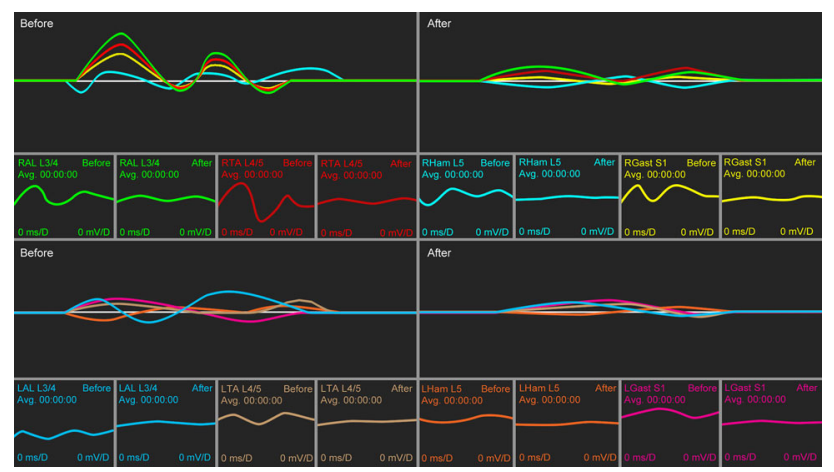

Fig. 4 Google Glass intraoperative display. $R A L, L 3 / 4$ right adductor longus, nerves L3-L4; RTA right tibialis anterior, nerves L4-L5; Rgast right gastrocnemius, nerve S1; Rham right medial hamstring, nerve L5. The same for the left side. Each amplitude wave correlates with a specific sensory nerve; these waves are identified via the use of colour. Green RAL L3/4, Red RTA L4/5, Turquoise RHam L5, Yellow RGast S1, Blue LAL L3/4, Brown LTA L4/5, Orange LHam L5, Purple LGast S1

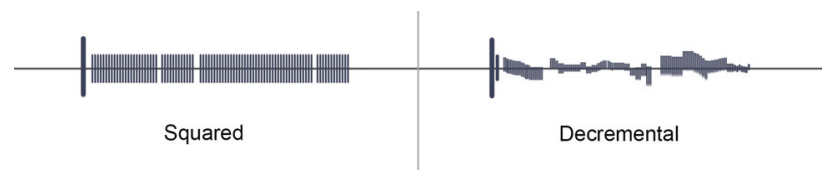

Fig. 5 Schematic diagram of normal intraoperative EMG responses

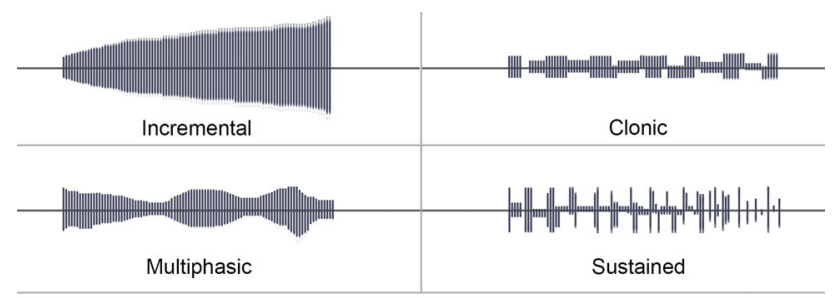

Stimulus: 1s Electrical train at $50 \mathrm{Hertz}$ Constant voltage at $0.1 \mathrm{~ms}$ duration

Fig. 6 Schematic diagram of abnormal intraoperative EMG responses

Figures 5 and 6 depict the patterns displayed from testing the nerve rootlets. Decremental and squared patterns are normal responses from EMG whereas all the responses on Fig. 5 are abnormal. A generic response is formed after a small incremental increase in voltage from stimulus intensities that previously evoked no responses. An irregular response is a fluctuation in EMG amplitude throughout the 1-s stimulus interval where the amplitude ratio of the highest-lowest amplitude was greater than or equal to 2. Of all these secondary abnormalities an abrupt response is considered to be most indicative of pathology,

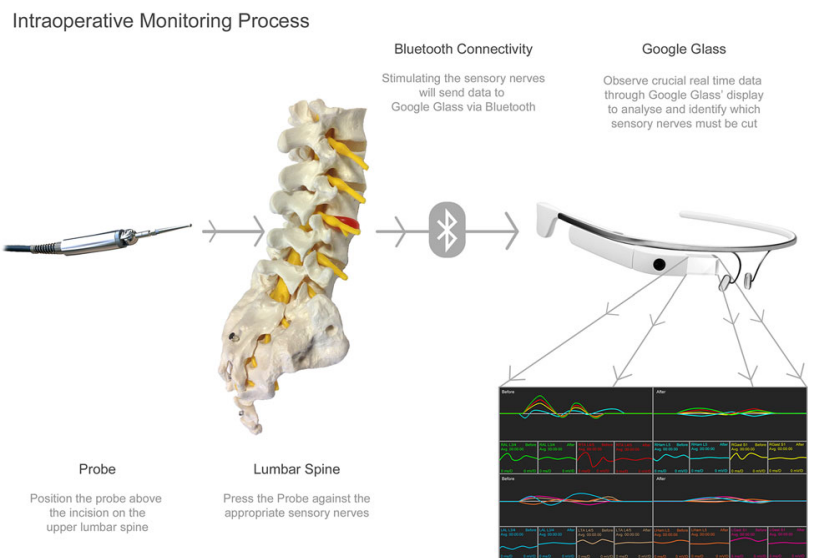

Fig. 7 Intraoperative monitoring process

whereas a low threshold is the least [12]. While testing the nerves the Neurophysiologist must determine whether the responses are normal or abnormal from the elicited waveforms. This procedure will be undertaken more than once and sometimes the same nerve will be tested more than once. Overall, SDR requires testing the rootlets with NIOM procedures and ablating the abnormal sensory roots that contribute to the spasticity. It is essential that the practitioner can see these waveforms during the procedure. Overlaying this information on Glass could improve the efficiency of this procedure and prevent potential loss of communication. While the practitioner is using EMG monitoring the surgeon can wear Glass and would be able to see the evoked potentials without having to take their focus away from the procedure. This could be very beneficial for the surgeon as they would be able to make an informed decision based on the data Glass provides in relation to which nerves to cut, rather than solely having to communicate with the Neurophysiologist across the operating theatre. It is also suggested that overlaying this data on Glass would improve the efficiency, safety and productivity of the SDR procedure. The information provided on the existing monitors would have to be re-designed specifically for Glass via information design, the display would need to be spatially and visually oriented to overlay and augment the working field of view.

Figure 7 depicts how Glass would function during the SDR procedure. Once a probe is pressed against the appropriate sensory nerves, Glass will project the data allowing a surgeon to make an informed decision in which nerves to cut.

Figures 8 and 9 show an updated design for Glass' (IONM) display. Presenting all the numerical values on one screen and the amplitude wave based data on another made the displays more intuitive. 


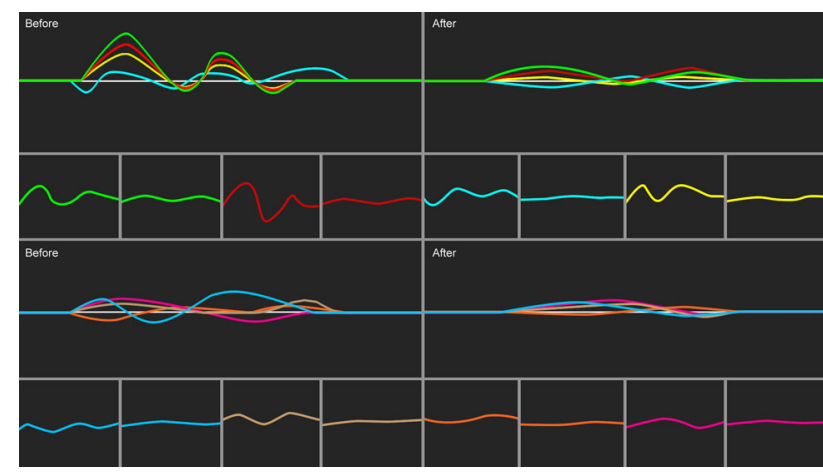

Fig. 8 Simplified intraoperative monitoring display

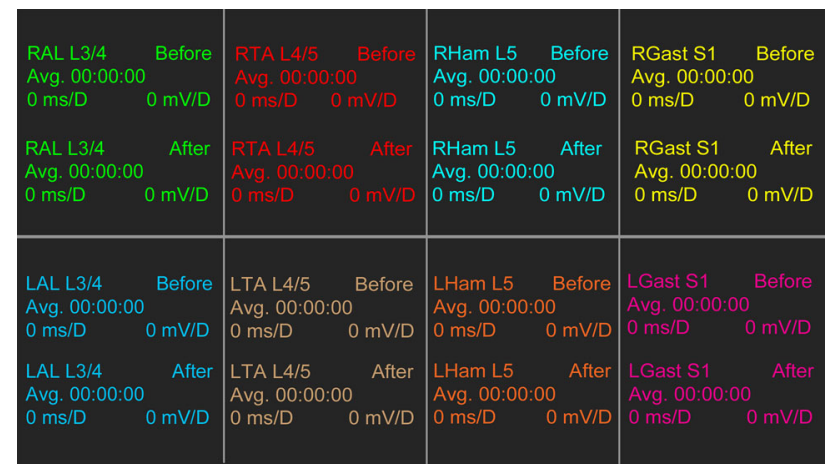

Fig. 9 Intraoperative monitoring display values

\section{Discussion}

\section{Future of Google Glass}

With the apparent failure of Glass within the consumer market Google decided to discontinue the sale of the device, but still provide the device to businesses. Looking ahead, Google realised that they had outgrown the lab, and so they are officially "graduating" from Google X [13]. With an official announcement from Google regarding the discontinuation of Glass this raised the question as to whether Glass was still suitable to be used for this project within the health care industry. However, it was decided that Glass was still fit for purpose and would be used as an example platform to present and illustrate the design and use of this device within the operating theatre. Alternative, wearable headsets such as the Microsoft HoloLens will also be examined for future development work. However, the HoloLens being essentially a powerful holographic computer raises the question as to whether this device is 'too immersive' and technically unwarranted for EMG monitoring. Google reported that further work on the future of the product will be undertaken but no specifications or designs have been disclosed to date. With a second iteration rumoured to arrive in 2016, it is reassuring that the original Google Glass device was suitable for this research.

\section{Discussion and conclusion}

This research proposes the design of an innovative wearable information display for monitoring electromyography responses within spinal surgery. Four key areas critically analyse the usefulness of a new intraoperative monitoring display design on an optical head-mounted display for monitoring electromyography responses:

1. It had been identified during the SDR procedure that the surgeon had to communicate with a Neurophysiologist across the operating theatre. This could result in a lack of communication, efficiency and the possibility of the surgeon over or under sectioning a particular nerve. The use of Glass in SDR would be able to help resolve this issue allowing the surgeon to view the EMG responses on the display.

2. The design process adopted gives this research a good structure, with original concepts devised on pre-made information design templates being the same size as Glass' display. Throughout the design and development process consultation with the surgeon validated design choices and provided critical analysis in order to create a fully functional and appropriate system design.

3. It was crucial that the display was intuitive and did not cause inattention blindness. The amplitude based waves were overlaid creating more space for additional features and making it easier for the surgeon to focus on important areas of the display. Keeping to traditional methods (amplitude waves) of analysing the data combined with contemporary methods (layout and colour) ensures that surgeons would not have to learn new techniques making this a simple but effective way of interpreting the required data. Additionally, the use of colour allows the user to distinguish which nerve and muscle relates to which amplitude wave. Compared to the existing intraoperative monitor this new layout is far more intuitive with an updated use of typography. The 'before and after' feature allows a surgeon to analyse the EMG responses before and after the sensory nerves are cut providing greater scope of how successful the procedure is. In addition rather than the surgeon seeing an EMG response every millisecond the new display would present an average EMG response every $10 \mathrm{~ms}$ allowing the surgeon to view a much less complicated version of the data.

4. Product testing and further consultation with the surgeon revealed that the display design met the 
required specifications and that Glass was fit for purpose.

Glass has successfully demonstrated its suitability as a platform to display the required information for the SDR procedure. Alternative wearable headsets could also be considered. Overall, further design development would allow Glass (Glass 2.0?) to be suitable for monitoring the appropriate surgical data.

\section{Compliance with ethical standards}

Conflict of interest The authors declare that they have no competing interests.

Open Access This article is distributed under the terms of the Creative Commons Attribution 4.0 International License (http://crea tivecommons.org/licenses/by/4.0/), which permits unrestricted use, distribution, and reproduction in any medium, provided you give appropriate credit to the original author(s) and the source, provide a link to the Creative Commons license, and indicate if changes were made.

\section{References}

1. Scope.org.uk Selective dorsal rhizotomy (SDR). http://www. scope.org.uk/support/families/therapies/sdr. Cited 30 Jun 2015

2. Gosh.nhs.uk (2012) Selective dorsal rhizotomylGreat Ormond Street Hospital. http://www.gosh.nhs.uk/medical-information/ procedures-and-treatments/selective-dorsal-rhizotomy. Cited 30 Jun 2015

3. Rouse M (2013) What is Google Glass/-definition from WhatIs.com. [Internet] Search Consumerisation. http://searchmo bilecomputing.techtarget.com/definition/Google-Glass. Cited 18 Jun 2015
4. Afshar V (2014) How Google Glass will transform healthcare. Huffington Post [internet]. http://www.huffingtonpost.com/valaafshar/how-google-glass-will-tra_b_6003100.html. Cited 19 Jun 2015

5. Glatter R (2014) Wearable technology and digital healthcare strategies should shift focus to chronic medical illness [Internet]. Forbes.com. Available from: http://www.forbes.com/sites/robert glatter/2014/11/20/wearable-technology-and-digital-healthcarestrategies-should-shift-focus-to-chronic-medical-illness/. Cited 20 June 2015

6. Iiid.net (2015) DefinitionsIIIID-International Institute for Information Design [Internet]. Available from: http://www.iiid. net/home/definitions/. Cited 7 July 2015

7. Stecker M (2012) A review of intraoperative monitoring for spinal surgery. Surg Neurol Int 3(4):174. Available from: http:// www.ncbi.nlm.nih.gov/pmc/articles/PMC3422092/. Cited 19 June 2015

8. Husain A (2008) Practical approach to neurophysiologic intraoperative monitoring. Demos Medical Publishing, New York, p 171

9. Husain A (2008) Practical approach to neurophysiologic intraoperative monitoring. Demos Medical Publishing, New York, p 169

10. Hammett TC, Boreham B, Quraishi NA, Mehdian SMH (2013) Intraoperative spinal cord monitoring during the surgical correction of scoliosis due to cerebral palsy and other neuromuscular disorders. Eur Spine J 22(S1):38-41. doi:10.1007/s00586-0122652-x

11. Husain A (2008) Practical approach to neurophysiologic intraoperative monitoring. Demos Medical Publishing, New York, p 172

12. Husain A (2008) Practical approach to neurophysiologic intraoperative monitoring. Demos Medical Publishing, New York, p 176

13. Plus.google.com (2015) We're graduating from Google[x] labIt's hard to believe that Glass started.... [Internet]. https://plus.goo gle.com/+GoogleGlass/posts/9uiwXY42tvc?e=-RedirectToSand box. Cited 16 August 2015 